\title{
ON THE GENERALISED TODD GENUS OF FLAG BUNDLES
}

\author{
by S. A. ILORI \\ (Received 14th February 1973)
}

\section{Introduction}

Let $V$ be a complex algebraic variety. Given integers $a_{1}, \ldots, a_{m}$ such that

$$
0 \leqq a_{1}<a_{2}<\ldots<a_{m}=n,
$$

one defines a $\left(a_{1}, \ldots, a_{m}\right)$-flag as a nested system

$$
S: S_{a_{1}} \subset S_{a_{2}} \subset \ldots \subset S_{a_{m}}, \operatorname{dim}_{c} S_{a_{i}}=a_{i},
$$

of subspaces of $S_{n}$, the $n$-dimensional complex projective space. The set of all such flags is called an incomplete flag-manifold in $S_{n}$, and is denoted by $W\left(a_{1}, \ldots, a_{m}\right)$. Also let $E$ be a complex $n$-dimensional vector bundle over $V$. Then we denote by $E\left(a_{1}, \ldots, a_{m-1}, n ; V\right)$ an associated fibre bundle of $E$ with fibre $W\left(a_{1}-1, \ldots, a_{m-1}-1, n-1\right) . \quad E\left(a_{1}, \ldots, a_{m-1}-1, n ; V\right)$ is called an incomplete flag bundle of $E$ over $V$ (cf. (2), (3)). In Section 10.3 and Section 14.4 of (1), the generalised Todd genus $T_{y}(W(0, n))$ and $T_{y}(W(0,1, \ldots, n))$ of the $n$-dimensional projective space $W(0, n)$ and the flag manifold $W(0,1, \ldots, n)$ (or $F(n+1)$ ) were calculated. Here we compute $T_{y}\left(W\left(a_{1}, \ldots, a_{m}\right)\right.$ ) and also $T_{y}\left(E\left(a_{1}, \ldots, a_{m-1}, \mathrm{n} ; V\right)\right)$.

Notation. We shall interpret the expression

to mean

$$
\sum_{i_{1}}^{n} t^{i_{1}} \sum_{i_{0}=0}^{i_{1}} t^{i_{0}}
$$

$$
1+t . \sum_{i_{0}=0}^{1} t^{i_{0}}+t^{2} . \sum_{i_{0}=0}^{2} t^{i_{0}}+\ldots+t^{n} . \sum_{i_{0}=0}^{n} t^{i_{0}} .
$$

By iteration, the expression

$$
\sum_{i_{m}=0}^{n} t^{i_{m}} \cdot \sum_{i_{m-1}=0}^{i_{m}} t^{i_{m-1}} \ldots \sum_{i_{0}=0}^{i_{1}} t^{i_{0}}
$$

will be interpreted similarly. We shall denote this last expression by

$$
\prod_{j=0}^{m} \sum_{i_{j}=0}^{i_{j+1}} t^{i_{j}}, \text { where } i_{m+1}=n .
$$




\section{Generalised Todd Genus}

\section{Lemma 2.1.}

$$
\frac{\left(1-t^{2(m+2)}\right)\left(1-t^{2(m+3)}\right) \ldots\left(1-t^{2(n+1)}\right)}{\left(1-t^{2}\right)\left(1-t^{4}\right) \ldots\left(1-t^{2(n-m)}\right)} \equiv \prod_{j=0}^{m} \sum_{i_{j}=0}^{i_{j+1}} t^{2 i_{j}},
$$

where $i_{m+1}=n-m$.

Proof. A simple inductive argument on $m$ shows that the right-hand side enumerates all partitions $\left(i_{0}, \ldots, i_{m}\right)$ such that

$$
0 \leqq i_{j} \leqq n-m \quad(j=0, \ldots, m) .
$$

But this is equal to the left-hand side from (7) and Section 26 of (6).

Corollary 2.2. The Poincaré polynomial of a Grassmannian is given by

where $i_{m+1}=n-m$.

$$
P(W(m, n): t)=\prod_{j=0}^{m} \sum_{i_{j}=0}^{i_{j+1}} t^{2 i_{j}}
$$

Proof. The proof follows immediately from the Lemma and from the formula of Hirsch (cf. (6)).

Theorem 2.3. The generalised Todd genus of $W\left(a_{1}, \ldots, a_{m-1}, n\right)$ is given by

where $a_{0}=-1, i_{a_{k}-a_{k-1}}=n-a_{k}$.

$$
T_{y}\left(W\left(a_{1}, \ldots, a_{m-1}, n\right)\right)=\prod_{k=1}^{m-1}\left[\prod_{j=0}^{a_{k}-a_{k}-1} \sum_{i_{j}=0}^{i_{j+1}}(-1)^{i_{j}} y^{i_{j}}\right] \text {, }
$$

Proof. We first prove the theorem in the case of a Grassmannian, $W(m, n)$. Consider the following flag bundles:

$$
F(n+1) \underset{F(n-m)}{\longrightarrow} W(0,1, \ldots, m, n) \underset{F(m+1)}{\longrightarrow} W(m, n) .
$$

From Section 14 of (1), the $T_{y}$-genus behaves multiplicatively with respect to flag bundles and so

$$
\begin{aligned}
T_{y}(W(m, n))= & \frac{T_{y}(F(n+1))}{T_{y}(F(m+1)) T_{y}(F(n-m))} \\
& =\frac{\left(1-y+y^{2}-\ldots+(-1)^{m+1} y^{m+1}\right) \ldots\left(1-y+y^{2}-\ldots+(-1)^{n} y^{n}\right)}{(1-y)\left(1-y+y^{2}\right) \ldots\left(1-y+y^{2}-\ldots+(-1)^{n-m-1} y^{n-m-1}\right)} .
\end{aligned}
$$

Poincaré polynomials also behave multiplicatively with respect to flag bundles (cf. (4)). Thus

$$
\begin{aligned}
P(W(m, n)) & =\frac{P(F(n+1))}{P(F(m+1)) \cdot P(F(n-m))} \\
& =\frac{\left(1+y^{2}+\ldots+y^{2(m+1)}\right) \ldots\left(1+y^{2}+\ldots+y^{2 n}\right)}{\left(1+y^{2}\right) \ldots\left(1+y^{2}+\ldots+y^{2(n-m)}\right)} .
\end{aligned}
$$


But from Lemma 2.1,

$$
P(W(m, n))=\prod_{j=0}^{m} \sum_{i_{j}=0}^{i_{j+1}} t^{2 i_{j}}, \text { where } i_{m+1}=n-m .
$$

Thus by comparing the various polynomials, we find that the generalised Todd genus of a Grassmannian is given by

$$
T_{y}(W(m, n))=\prod_{j=0}^{m} \sum_{i_{j}=0}^{i_{j+1}}(-1)^{i_{j} y^{i_{j}}}, \quad \text { where } i_{m+1}=n-m .
$$

Now to find the generalised Todd genus of $W\left(a_{1}, \ldots, a_{m-1}, n\right)$, we consider the following sequence of flag bundles:

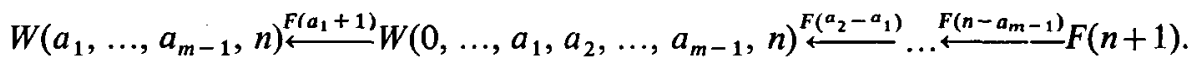

Hence

$$
\begin{gathered}
T_{y}\left(W\left(a_{1}, \ldots, a_{m-1}, n\right)\right)=\frac{T_{y}(F(n+1))}{T_{y}\left(F\left(a_{1}+1\right)\right) T_{y}\left(F\left(a_{2}-a_{1}\right)\right) \ldots T_{y}\left(F\left(n-a_{m-1}\right)\right)} \\
=\frac{T_{y}(F(n+1))}{T_{y}\left(F\left(a_{1}+1\right)\right) \cdot T_{y}\left(F\left(n-a_{1}\right)\right)} \cdots \frac{T_{v}\left(F\left(n-a_{n-2}\right)\right)}{T_{y}\left(F\left(a_{m-1}-a_{m-2}\right)\right) \cdot T_{y}\left(F\left(n-a_{m-1}\right)\right)} \\
=T_{y}\left(W\left(a_{1}, n\right)\right) \cdot T_{y}\left(W\left(a_{2}-a_{1}-1, n-a_{1}-1\right)\right) . \\
\quad \ldots T_{y}\left(W\left(a_{m-1}-a_{m-2}-1, n-a_{m-2}-1\right)\right) .
\end{gathered}
$$

The theorem now follows from (2.4).

Corollary 2.5. The Todd genus of all flag manifolds is equal to 1, i.e.

$$
T\left(W\left(a_{1}, \ldots, a_{m-1}, n\right)\right)=1 .
$$

Proof. Put $y=0$ in the theorem since for a variety $V$,

$$
T(V)=T_{0}(V) \text { (cf. Section } 10 \text { of (1)). }
$$

Corollary 2.6. $T_{y}\left(E\left(a_{1}, \ldots, a_{m-1}, n ; V\right)\right)=T_{y}(V) . T_{y}\left(W\left(a_{1}-1, \ldots, n-1\right)\right)$.

Proof. $E\left(a_{1}, \ldots, a_{m-1}, n ; V\right) \rightarrow V$ is a fibre bundle, fibre

$$
W\left(a_{1}-1, \ldots, a_{m-1}-1, n-1\right) \text {. }
$$

From Section 14 of (1) and the theorem it follows that the $T_{y}$-genus behaves multiplicatively with respect to incomplete flag bundles and so the corollary follows.

Corollary 2.7. $T\left(E\left(a_{1}, \ldots, a_{m-1}, n ; V\right)\right)=T(V)$.

Proof. Put $y=0$ in Corollary 2.6 and use Corollary 2.5.

\section{REFERENCES}

(1) F. Hirzebruch. Topological Methods in Algebraic Geometry, 3rd edition (Springer, Berlin, 1966). 
(2) A. W. INgLeton, Tangent flag bundles and generalized Jacobian varieties, Rend. Accad. dei Lincei (1969), 323-329, 505-510.

(3) Séminaire Chevalley (2) 1958, Anneaux de Chow et applications.

(4) S. S. Chern, F. Hirzebruch and J.-P. Serre, On the index of a fibered manifold. Proc. Amer. Math. Soc. 8 (1957), 587-596.

(5) B. L. VAN Der W Aerden, Modern Algebra Vol. I and II (Ungar, New York, 1949).

(6) A. Borel, Sur la cohomologie des espaces fibrés principaux et des espaces homogènes de groupes de Lie compacts, Ann. of Math. (2) 57 (1953), 115-207.

(7) C. Ehresmann, Sur la topologie de certains espaces homogènes, Ann. of Math. (2) 35 (1934), 396-443.

UNIVERSITY OF IBADAN

NIGERIA 